\title{
Nanostructured Lipid Carrier (NLC) and Solid Lipid Nanoparticles (SLN) Based Hydrogel for Delivery of Etodolac: A Comparative Investigation on Dermal Pharmacokinetic on Rat Skin
}

\author{
Dilip Kumar Patel*, Roohi Kesharwani, Vikas Kumar \\ Department of Pharmaceutical Sciences, Sam Higginbottom University of Agriculture, Technology and Sciences, Naini, Prayagraj, Uttar Pradesh, INDIA.
}

\begin{abstract}
Objectives: The present work focus on to create Solid lipid nanoparticles (SLN) and nanostructured lipid Carrier (NLC) as transporters for effective delivery of Etodolac (ETD). Methods: Etodolac stacked SLN and NLC were set up by melting emulsification and hardening at low temperature method and they were portrayed for molecule size, zeta potential, encapsulation efficacy and in vitro discharge rate. Ex vivo dermatokinetic by tape stripping technique was explored in rat skin utilizing altered Franz diffusion cells. The praticle size and the surface electrical charge (ZP) have been assessed following after manufacturing of dispersion. Results: The particles stayed in their colloidal range after production by melt emulsification method. For all nano dispersion the encapsulation efficacy was higher than $75 \%$. Differential Scanning calorimetry (DSC) were performed to portray the physicochemical properties of the SLN and NLC. Approximately Same amount of Etodolac release was facilitated through skin of rats from SLN-SA and NLC-SA. Conclusion: Research work could be concluded as
\end{abstract}

successful development of Etodolac loaded SLN and NLC to increase the encapsulation efficiency of colloidal lipid carriers with benefit of higher performance in terms of long term stability and impart a sustaining Etodolac topical effect as well as faster relief from pain associated with arthritis.

Key words: Etodolac, Nanostructure lipid carrier, NLC, Solid Lipid Nanoparticles, SLN, Topical gel.

\section{Correspondence}

Mr. Dilip K Patel

Department of Pharmaceutical Sciences, Sam Higginbottom University of Agriculture, Technology and Sciences, Naini, Prayagraj, Uttar Pradesh, INDIA.

Email: dilippatel87mph@gmail.com

DOI: 10.5530/ijpi.2021.2.39

\section{INTRODUCTION}

Solid Lipid Nanoparticles (SLN) were developed in the end of $20^{\text {th }}$ century they owns various highlights beneficial topically. Few potential problems can occur depending on the API, for example insufficient total drug load and leakage of drug occurs throughout storage. ${ }^{1-3}$ To control the drawbacks associated with SLN the Nanostructured lipid carriers (NLCs) have been evolved. The NLCs comprises high content of liquid lipid too with solid lipid matrix. Lately, colloidal dispersions produced using combination of solid and liquid lipids contribute to controlled release characteristics with higher drug loading capacities in comparison to SLN. It has been recommended that these NLC are comprised of oily droplets that helps in solubilisation of drug. ${ }^{4-6}$

Etodolac is an exceptionally compelling non-steroidal antiinflammatory, pain relieving moiety and utilized against rheumatoid joint inflammation. The GI system is affected by some unfavorable adverse effects and physicochemical properties associated with it such as poor solubility, hence having less utilization in therapeutic index. Drug (etodolac) restrain the chemical that makes prostaglandins (cyclooxygenase), bringing about let down concentration. Result in decrees in pain associated with inflammation and fever. ${ }^{7-10}$ The main objective of this study was to assess and evaluate the comparative study between SLN and NLC on the skin obstruction properties via ex-vivo permeation study. It is normal that the outcomes acquired add to the comprehension of these lipid-based systems to enhance their therapeutic performance.

\section{MATERIALS AND METHODS}

\section{Materials}

Stearic acid, Lecithin was purchased from Himedia Mumbai India, Eucalyptus oil was purchased from Loba Chemie India, carbopol and carrageenan were kindly supplied by Guapha pharmaceutical India and The API (Etodolac) was kindly gifted by Lupin India,. All the other chemicals were of analytical grade.

\section{Preparation of SLN and NLC dispersion}

The melt emulsification and solidification at low temperature technique were used for the preparation of SLN (containing Stearic Acid) and NLC (composed of Stearic Acid and Eucalyptus oil 70:30) (Table 1). Briefly, a hot $2.0 \%$ Tween 80 solution was heated at $90^{\circ} \mathrm{C}$ and another phase containing lipid (solid lipid or lipids blend), Etodolac and lecithin premixed and heated above melting point. The resultant heated mixture is added drop wise to surfactant solution overhead stirrer to obtain a clear emulsion. The dispersion formed was moved into a cold icy water bath i.e. at $\left(-2^{\circ} \mathrm{C}\right)$ with continuous stirring for $2 \mathrm{hr}$ at $5000 \mathrm{rpm}$. By solidifying of the lipid beads present in the emulsions the lipid nano dispersion (SLN and NLC) are formed. The prepared dispersion was kept in cool temperature for additional analysis. ${ }^{11}$

\section{SLN and NLC Dispersion Characterization}

Malvern Mastersizer 2000MU (Malvern instrument Great Britain with a minimum detection limit $10 \mathrm{~nm}$ ) were used to determine particle by utilizing Laser diffraction technique. For the determination of zeta 
Table 1: Composition of SLN and NLC topical gel.

\begin{tabular}{ccccccc}
\hline S.No. & System & $\begin{array}{c}\text { Stearic Acid } \\
(\mathbf{m g})\end{array}$ & $\begin{array}{c}\text { Eucalyptus oil } \\
(\mathbf{m g})\end{array}$ & $\begin{array}{c}\text { Etodolac } \\
(\mathbf{m g})\end{array}$ & $\begin{array}{c}\text { Lecithin } \\
(\mathbf{m g})\end{array}$ & $\begin{array}{c}\text { Tween } 80 \\
\% w / v\end{array}$ \\
\hline 1 & SLN-SA-Blank & 1000 & -- & -- & 100 & 2.0 \\
2 & SLN-SA-ETD & 1000 & -- & 100 & 100 & 2.0 \\
3 & NLC-SA-Blank & 700 & 300 & -- & 100 & 2.0 \\
4 & NLC-SA-ETD & 700 & 300 & 100 & 100 & 2.0 \\
\hline
\end{tabular}

potential a photon correlation spectroscopy technique is used with the help of Malvern Zetasizer ${ }^{\circledR}$ Nano-ZS (Malvern Instruments, USA). For this purpose the samples were diluted with deionized water and it was estimated by considering viscosity, dielectric constant and refraction index of water at $25^{\circ} \mathrm{C}(n=5)$. The scanning electron microscopy (SEM) is utilized to assessed the morphology of the lipid nanoparticles using a ZEISS Multi SEM 505, Germany.

\section{Etodolac Entrapment and Loading Capacity}

By examining the concentration of free ETD in the dispersion medium a certain rate the Entrapment efficacy EE (\%) of nanoparticles was calculated. The SLN sedimented by centrifugation of nano dispersion containing Etodolac (2ml) for $45 \mathrm{~min}$ at $12500 \mathrm{rpm}$ (Remi Motor India). The dilution of collected supernatant was done with methyl alcohol and the sample were evaluated using a spectrophotometer (UV-1800 Shimadzu Japan) at $278 \mathrm{~nm}$. The entrapment efficiency percentage was calculated as follows:

$\mathrm{EE}=\left(\frac{\mathrm{Wa}-\mathrm{Ws}}{\mathrm{Wa}}\right) \times 100$

Where,

$\mathrm{EE}=$ entrapment efficiency,

$\mathrm{Wa}=$ mass of Etodolac added to the formulation,

Ws = analyzed weight of the drug in supernatant

$\mathrm{W} l=$ weight of lipid added

\section{Differential scanning calorimetry (DSC)}

The crystallization behaviour of lipid and drug-lipid interaction were analysed by DSC (pure lipid, placebo and drug loaded nanoparticles). For the purpose of DSC analysis the sample $(5 \mathrm{mg}$ ) were heated in aluminum pan between $10-400^{\circ} \mathrm{C}$ (DSC METTELER, Switzerland) at a rate of $10^{\circ} \mathrm{C} / \mathrm{min}$ under a nitrogen flow rate of $20 \mathrm{ml} / \mathrm{min}$. The Differential scanning analysis were performed for the following sample, Etodolac (ETD), stearic acid (SA), mixture of solid lipid and Etodolac (SA+ETD) SLN and NLC of etodolac and mixture of stearic acid and eucalyptus oil.

\section{Formulation and evaluation of Etodolac loaded SLN and NLC gel}

Carbopol was utilized for the preparation of gel; it was dispersed in the SLN and NLC dispersion utilizing a mechanical stirrer (Remi, Mumbai, India) for this purpose with speed of $1200 \mathrm{rpm}$ and for the neutralization of dispersion the triethanolamine is used. The gel was analyzed for the spreadibility for this purpose the $1.0 \mathrm{~g}$ of nano gel kept in between two glass plate $1.0 \mathrm{~cm}^{2}$. By applying weight of $50 \mathrm{~g}$ for $5 \mathrm{~min}$ the diameter of the gel sample increases due to spreading of nano gel and diameter were noted down. ${ }^{12}$

\section{In vitro release of Etodolac from SLN and NLC Gel}

The in vitro release of etodolac from prepared SLN and NLC formulation was evaluated by using Modified Franz diffusion cell (Zenith Glassware India). In this the membrane (Dialysis membrane $70 \mathrm{Hi}$-Media, India) with pore size $2.4 \mathrm{~nm}$, with surface area of membrane $3.14 \mathrm{~cm}^{2}$ was utilized. The receptor compartment of diffusion assembly was loaded

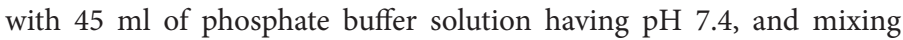
accomplished by using magnetic stirrer at $750 \mathrm{rpm}$ at $37^{\circ} \mathrm{C} \pm 0.5^{\circ} \mathrm{C}$. The donor compartment containing sample (SLN gel) the sample was collected from the receptor compartment at specific interval and exact similar quantity of phosphate buffer solution was added in it. Lastly the samples were diluted and investigated for release pattern of formulation by using spectrophotometer at $278 \mathrm{~nm} \cdot{ }^{13,14}$

\section{Dermal pharmacokinetic studies}

This study was carried to assess the amount of etodolac held in skin by utilizing tape stripping technique with Scotch Magic Tape. ${ }^{12,15,16}$ For this purpose the gel sample was applied to the skin of rat and impeded for 24 $\mathrm{hr}$. After $24 \mathrm{hr}$ the residuals were swipe with the help of cotton, followed by washing and air drying. This cycle is repeated for ten times, as the primary tape strip was set for clearing the Stratum corneum of rat skin and so on repeated for same. After that the skin was homogenized by methanol and centrifuged for $10 \mathrm{~min}$ at $7000 \mathrm{rpm}$. Lastly the amount of drug retained in skin was calculated by utilizing HPLC technique (Sys LC-138, Systronic India). ${ }^{17}$

\section{Analysis of ETD by High Performance Liquid Chromatography (HPLC)}

The drug (Etodolac) analyzed by HPLC technique, in this the Acetonitrile: water (50:50) contained by mobile phase and the $\mathrm{pH}$ adjusted to 5.8 with orthophosphoric acid. The ultrasonication was done for formerly filtered mobile phase for $15 \mathrm{~min}$. by utilizing $0.45 \mu$ membrane with flow rate 1.0 $\mathrm{ml} / \mathrm{min}$. It was analysed at constant column temperature $\left(25 \pm 2^{\circ} \mathrm{C}\right)$ at wavelength $278.0 \mathrm{~nm} .^{8}$

\section{Statistical Analysis}

Graph Pad Prism software was utilized for the statistical analysis of data obtained from in vitro drug release and drug penetration studies through excised rat skin and one-way ANOVA is helpful to calculate differences between profiles. The level of significance was set as $P<0.05$.

\section{RESULTS}

\section{Lipid Nanoparticle (SLN and NLC) Characterization}

The Table 2 depicted particle size distribution values of dispersion (SLN and NLC). The SLN-SA-ETD shows particle size distribution d90\% was $518 \mathrm{~nm}$ where as for NLC-SA-ETD it was $304 \mathrm{~nm}$ (Figure 1). The long term physical stability of dispersion ensured via assessment of zeta potential. So in this the calculated value of zeta it was observed that the value higher than-30 $\mathrm{mV}$ show better stability. The optimized zeta 
potential of formulation ought to be around $-60 \mathrm{mV}$ for better shelf life. The zeta potential value of SLN-SA-ETD and NLC-SA-ETD was found to be $-21.2 \mathrm{mV}$ and $-21.60 \mathrm{mV}$ in our research. ${ }^{9,18}$

\section{Scanning Electron Microscopy}

The morphology of lipid nanoparticles (SLN and NLC) showed by scanning electron microscopy (SEM) images as indicated in Figure 2. Since the two systems i.e. SLN and NLC dispalay comparable qualities they prepared under similar condition with same main lipid. ${ }^{19,13}$

\section{Differential scanning calorimetric study}

In this for the investigation of DSC the heating of bulk lipid (Stearic Acid) bring about thermal transitions. DSC curve of Stearic acid revealed sharp endothermic peak at $62.06^{\circ} \mathrm{C}$ accompanying the melting point of lipid as depicted in (Table 3 ). The onset value of solid lipid was showed as $56.97^{\circ} \mathrm{C}$ and the theoretical melting enthalpy was found to be $-864.68 \mathrm{~mJ}$.
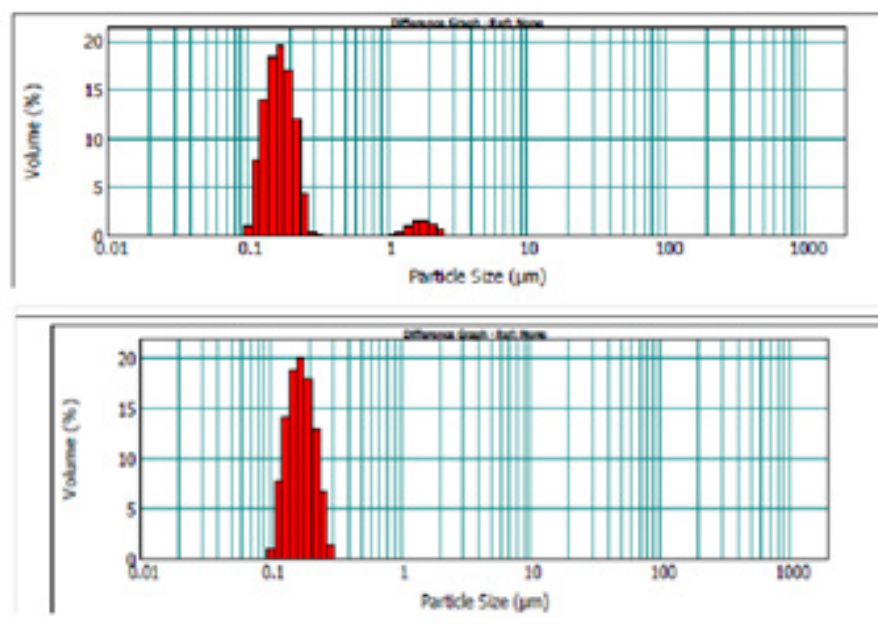

Figure 1: Particle size of formulation SLN-SA and NLC-SA-by Malvern mastersizer.

Table 2: Particle size, Entrapment efficacy and Zeta potential of formulation.

\begin{tabular}{ccccc}
\hline S.No. & System & $\mathrm{d} 90 \% *$ & $\%$ EE* $^{*}$ & $\begin{array}{c}\text { Zeta } \\
\text { Potential* }\end{array}$ \\
\hline 1 & SLN-SA-Blank & $613 \pm 10.67$ & -- & $-22.8 \pm 0.570$ \\
2 & SLN-SA-ETD & $518 \pm 14.67$ & $76.01 \pm 0.652$ & $-21.2 \pm 0.245$ \\
3 & NLC-SA-Blank & $380 \pm 2.64$ & -- & $-22.20 \pm 0.30$ \\
4 & NLC-SA-ETD & $304 \pm 1.56$ & $83.73 \pm 064$ & $-21.60 \pm 0.42$ \\
\hline
\end{tabular}

${ }^{*} n=3$

Table 3: DSC curve data of thermograme.

\begin{tabular}{ccccc}
\hline Thermograme & Enthalpy* & $\begin{array}{c}\text { Onset } \\
\text { point* }\end{array}$ & Peak point* & $\begin{array}{c}\text { Endset } \\
\text { point* }\end{array}$ \\
\hline Etodolac & $-208.84 \mathrm{~mJ}$ & 152.19 & 155.43 & 160.36 \\
Stearic acid & $-864.68 \mathrm{~mJ}$ & 56.97 & 62.06 & 69.31 \\
SLN of Etodolac & $-628.05 \mathrm{~mJ}$ & 56.57 & 61.58 & 67.93 \\
NLC of Etodolac & $-363.85 \mathrm{~mJ}$ & 54.24 & 57.84 & 61.81 \\
\hline
\end{tabular}

${ }^{*} n=3$

\section{Evaluation of Etodolac based SLN gel}

The SLN and NLC based gel containing etodolac was prepared by using $1.5 \% \mathrm{w} / \mathrm{w}$ of Carbopol 930 to the nano dispersion at last achieved translucent appearance. It was observed that as increase in weight the spreadability of the gel is increased, which suggests that on rubbing the gel onto the skin spreadability of the gel can be enhanced. It was also observed that spreadability of blank gel was found to be lesser (60.8 $\mathrm{cm})$ as compare to SLN and NLC based gel $(6.86 \mathrm{~cm})$ which might be ascribed to increased solid content of the gel after addition of lipid nanoparticulates.

\section{In vitro release study of SLN gel}

The in vitro release study helps to assess the drug (Etodolac) release profile Etodolac loaded SLN and NLC gel. The process accomplished in $24 \mathrm{hrs}$ to obtain cumulative percent release of drug from SLN and NLC formulation and each sample was evaluated three times. The release profile of etodolac from nano gel, Formulation SLN-SA Figure 4 depicted as 16.24 percent release in the initial $15 \mathrm{~min}$ and in case of NLC-SA gel it was depicted as 20.84 percent it may be due to depression in melting point of lipid matrix due to presence of liquid lipid (eucalyptus oil).
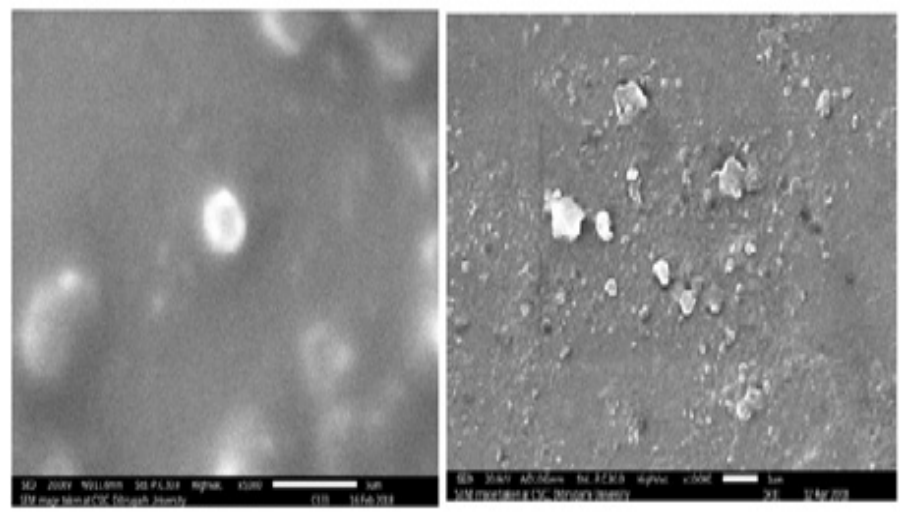

Figure 2: SLN-SA and NLC-SA Dispersion SEM Image.

\section{Skin retention study by tape stripping method}

This study was performed via tape stripping method and it was observed that in SLN gel the retention of amount of drug (ETD) is $31 \pm 0.68$ microgram per centimeter square and $30.06 \pm 0.45$ microgram per centimeter square with NLC gel.

\section{DISCUSSION}

SLNs and NLC dispersion were manufactured via utilising modified technique (Melt Emulsification and solidification at low temperature method). It's also offers economical and easy process as contrast to various technique like Easy of handling, easy and potent method, not utilisation of excess organic solvents and energy during the manufacturing of SLN and NLC dispersion. The particle diameter of NLC is lower as compare to SLN because it contain eucalyptus oil $30 \%$ in it solid lipid blend. In this it was observed that the decrease in electrical charge at the surface of both system (SLN and NLC) due to incorporation of drug (ETD). When looking at the two systems, NLC additionally revealed lower ZP than the individual SLN. These outcomes could be taken as a sign that ETD is entrapped by the lipid matrix of SLN 
and NLC system. SEM study revealed that the particles were of spherical shape with smooth and nonporous surface. The spherical structure of particles concurs with the outcomes got from the instrument Malvern mastersizer. It was observed bunch of particles inferable from the carrier's polymorphic presence in SEM picture. In this few of them do not have a spherical shape inferable from the polymorphism of the lipid in the process of heating. The concentration of the liquid lipid play a major role as it increases at a constant amount of lipid the surface of the formed NLCs is smaller which absorb Tween 80 surfactant molecules which could lead to the synthesis of the drug's surfactant Solution. Hence the Etodolac solubility was enhanced in aqueous phase as well as reduces drug leaching. The entrapment in SLN formulation is lesser because of it does not contain liquid lipid. Percent entrapment is higher in case of NLC dispersion due to presence of eucalyptus oil which cause imperfection

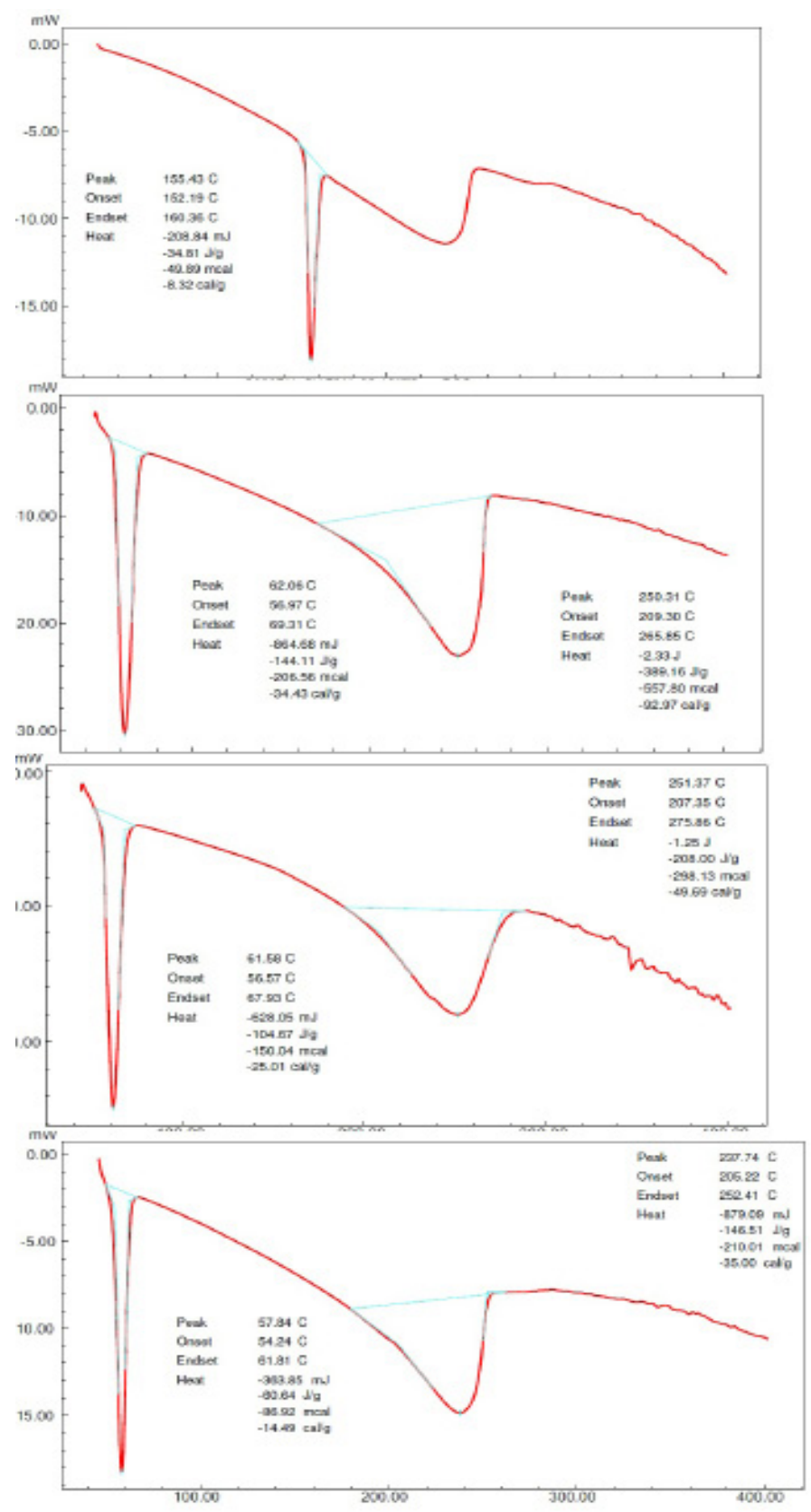

Figure 3: DSC Curve of Etodolac, Stearic acid, SLN, and NLC Formulation.

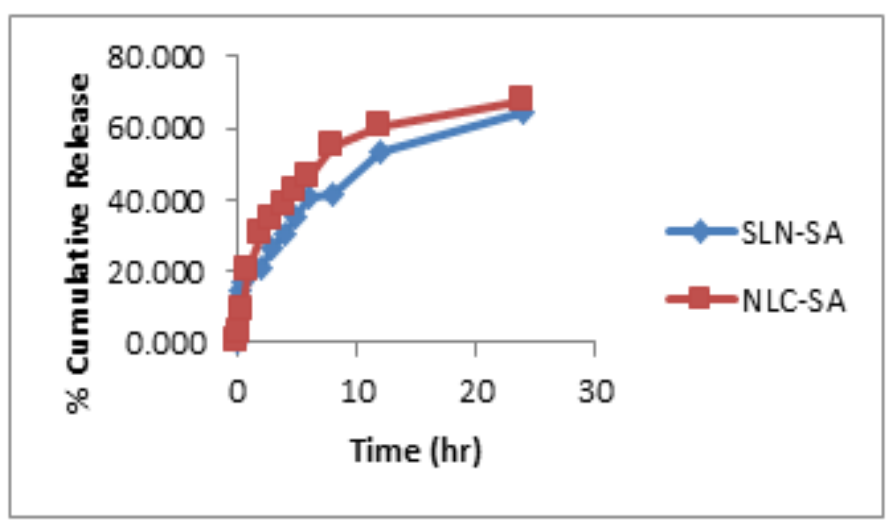

Figure 4: Percentage cumulative release of SLN and NLC based gel.

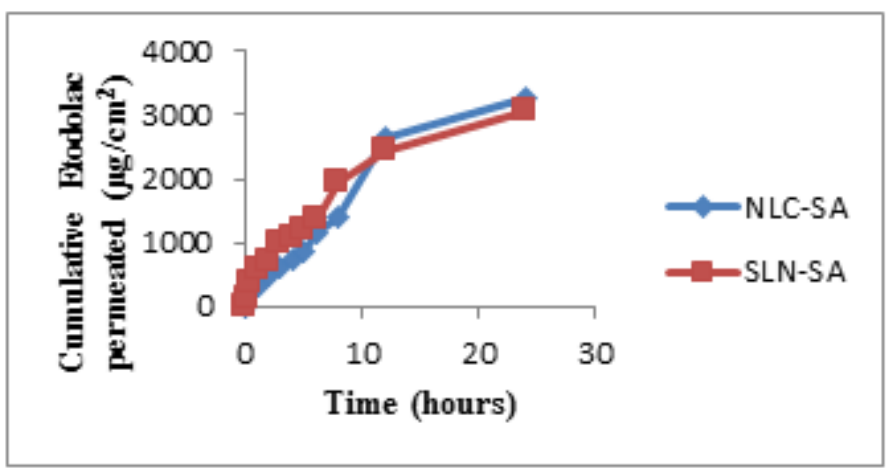

Figure 5: Cumulative Etodolac permeated vs Time of SLN and NLC based gel.

in crystal lattice of lipid particle. As per information gathered by DSC experiments on formulations, melting points of nanoparticles were distinguished to diminish to lower temperatures in conjunction with broader peaks contrasted with bulk lipid by giving variable enthalpy values additionally showing several thermal transitions..$^{20,21}$ The melting points of the lipid only shown at $61.58^{\circ} \mathrm{C}$ and $57.84^{\circ} \mathrm{C}$ (enthalpy of -628.05 and $-363.85 \mathrm{~mJ}$ ) through DSC analysis of the SLN and NLC formulation respectively this implies that the drug is obviously dissolved in the lipid and dispersed in the lipid phase molecularly (Figure 3). It was also observed a depression in melting point of NLC due to presence of liquid lipid (Eucalyptus oil). The spreading diameter of SLN and NLC gel was found to be $06.68 \mathrm{~cm}$ and $06.52 \mathrm{~cm}$ individually demonstrating as solid spreadability. The amount of burst release of the drug from both nano dispersion was quite different. The concentration of burst release has a direct relation to the amount of etodolac that present on the SLN and NLC outer surface. Greater the burst release of Etodolac higher the permeation of drug through the membrane. Etodolac release was the minimal with SLN in comparison to NLC as depicted in Figure 5. Etodolac release was facilitated through skin of rats from NLC-SA shows higher amount i.e. (135.619 ug/ $\left.\mathrm{cm}^{2} / \mathrm{hr}\right)$ in comparison to SLNSA $\left(127.027 \mathrm{ug} / \mathrm{cm}^{2} / \mathrm{hr}\right)$ as depicted in Figure 5. There is more in skin aggregation was seen which shows the more polar nature of etodolac and the suitable action of lipid nanoparticle on rat skin (Occulsive effect). Neverthless the negligible difference was acquired between etodolac permeation profiles of SLN and NLC.

\section{CONCLUSION}

In this investigation, Solid lipid nanoparticle and Nanostructure lipid carrier frameworks can be considered as advanced frameworks for 
effective delivery of Etodolac. Stable SLN-SA and NLC-SA were set up by melt emulsification and hardening at low temperature strategy. The particle size of both (SLN and NLC) dispersion was in colloidal range. The entrapment efficacy and the Etodolac release profile depend upon the amount and the lipid combination used (Stearic acid and eucalyptus oil). NLC show higher entrapment because of their eucalyptus oil part. In consent to this outcomes NLC likewise show a faster delivery profile in contrast with SLN with a similar lipid focus. The acquired outcomes mirror the capability of SLN and NLC as a carrier for skin delivery of Etodolac which is exhibiting higher Etodolac penetration into skin. The acquired outcomes additionally showed the utilization of these lipid nanoparticles as altered delivery system for lipophilic medications.

\section{ACKNOWLEDGEMENT}

We are very much thankful to University Grant Commission (UGC) for their financial support (NFOBC-JRF) for this work.

\section{CONFLICT OF INTEREST}

The authors declare that there is no conflict of interest.

\section{ABBREVIATIONS}

ETD: Etodolac; SLN: Solid Lipid Nanoparticle; NLC: Nanostructuctured Lipid Carrier; API: Active Pharmaceutical Ingredient; ZP: Zeta Potential; DSC: Differential Scanning Calorimetry; EE: Entrapment Efficiency; SA: Stearic Acid; HPLC: High Performance Liquid Chromatography; ANOVA: Analysis of Variance; SEM- Scanning Electron Microscopy.

\section{REFERENCES}

1. Mehnert W, Mader K. Solid lipid nanoparticles: Production, characterization and applications. Adv Drug Deliv Rev. 2001. doi:S0169-409X(01)00105-3 [pii]

2. Patel DK, Kesharwani R, Kumar V. Lipid Nanoparticle Topical and Transdermal Delivery: A Review on Production, Penetration Mechanism to Skin. Int J Pharm Investig. 2019;9(4):148-53. doi:10.5530/ijpi.2019.4.28

3. Patel D, Kumar V, Kesharwani R, Mazumdar B. Lipid nanoparticle a novel carrier for cosmetics and topical preparation: A review. Inven Rapid Cosmeceuticals. 2015;1-6.

4. Radtke M, Müller R. Nanostructured lipid drug carriers. New Drugs. 2001.

5. Patel DK, Tripathy S, Nair SK, Kesharwani R. Nanostructured lipid carrier (NLC) a modern approach for topical delivery: A review. World J Pharm Pharma Sci. 2013;2:921-38

6. Müller RH, Petersen RD, Hommoss A, Pardeike J. Nanostructured lipid carriers (NLC) in cosmetic dermal products. Adv Drug Deliv Rev. 2007;59(6):522-30. doi:10.1016/j.addr.2007.04.012
7. Saleh OA, El-Azzouny AA, Aboul-Enein HY, Badawey AM, Rashed MS. Development and validation of stability-indicating high performance liquid chromatographic (HPLC) and DD1-spectrophotometric assays for etodolac in bulk form and in pharmaceutical dosage form. J Liq Chromatogr Relat Technol. 2009. doi:10.1080/10826070903249799

8. Krishna SRV, Belemkar S, Tiwari RN. RP-HPLC method development and validation of etodolac and paracetamol in tablet dosage form. Int J PharmTech Res. 2014;6(2):775-82

9. Asthana SG, Asthana A, Singh D, Sharma PK. Etodolac Containing Topical Niosomal Gel: Formulation Development and Evaluation. J Drug Deliv. 2016. doi:10.1155/2016/9324567

10. Patel DK, Kesharwani R, Kumar V. Etodolac loaded solid lipid nanoparticle based topical gel for enhanced skin delivery. Biocatal Agric Biotechnol. 2020. doi:10.1016/j.bcab.2020.101810

11. Kakkar V, Muppu SK, Chopra K, Kaur IP. Curcumin loaded solid lipid nanoparticles: An efficient formulation approach for cerebral ischemic reperfusion injury in rats. Eur J Pharm Biopharm. 2013;85(3):339-45. doi:10.1016/j.ejpb.2013.02.005

12. Mohammadi-Samani S, Zojaji S, Entezar-Almahdi E. Piroxicam loaded solid lipid nanoparticles for topical delivery: Preparation, characterization and in vitro permeation assessment. J Drug Deliv Sci Technol. 2018;47:427-33. doi:10.1016/j. jddst.2018.07.015

13. Kesharwani R, Sachan A, Singh S, Patel D. Formulation and Evaluation of Solid Lipid Nanoparticle (SLN) Based Topical Gel of Etoricoxib. J Appl Pharm Sci. 2016;6(10):124-131. doi:10.7324/JAPS.2016.601017

14. Patel D, Dasgupta S, Dey S, Ramani RY, Ray S, Mazumder B. Nanostructured lipid carriers (NLC)-based gel for the topical delivery of aceclofenac: Preparation, characterization, and in vivo evaluation. Sci Pharm. 2012;80(3):749-64. doi:10.3797/scipharm.1202-12

15. Wissing SA, Müller $\mathrm{RH}$. The influence of the crystallinity of lipid nanoparticles on their occlusive properties. Int J Pharm. 2002;242(1-2):377-9. doi:10.1016/ S0378-5173(02)00220-X

16. Jayaraman SC, Ramachandran C, Weiner N. Topical delivery of erythromycin from various formulations: An in vivo hairless mouse study. J Pharm Sci. 1996;85(10):1082-4. doi:10.1021/js960040u

17. Sanap GS, Mohanta GP. Development of miconazole nitrate controlled release formulations based on sln and NLC for topical delivery. Int J Pharm Pharm Sci. 2014;6(4):393-9

18. Uprit S, Sahu RK, Roy A, Pare A. Preparation and characterization of minoxidil loaded nanostructured lipid carrier gel for effective treatment of alopecia. Saud Pharm J. 2013:21(4):379-85. doi:10.1016/j.jsps.2012.11.005

19. TeeranachaideekulV, Müller RH, Junyaprasert VB. Encapsulation of ascorbyl palmitate in nanostructured lipid carriers (NLC)-Effects of formulation parameters on physicochemical stability. Int J Pharm. 2007:340(1-2):198-206. doi:10.1016/j. ijpharm.2007.03.022

20. Üner M, Karaman EF, Aydoğmus Z. Solid lipid nanoparticles and nanostructured lipid carriers of loratadine for topical application: Physicochemical stability and drug penetration through rat skin. Trop J Pharm Res. 2014;13(5):653-60. doi:10.4314/tjpr.v13i5.1

21. Üner M, Yener G, Erguven M, Karaman E, Utku E. Solid Lipid Nanoparticles and Nanostructured Lipid Carriers of Celecoxib for Topical Application - Preparation, Characterization and Drug Penetration Through Rat Skin. Curr Nanosci. 2014;10(4):532-42. doi:10.2174/1573413710666140218231307.

Article History: Submission Date : 02-04-2021; Revised Date : 21-05-2021; Acceptance Date : 18-06-2021.

Cite this article: Patel DK, Kesharwani R, Kumar V. Nanostructured Lipid Carrier (NLC) and Solid Lipid Nanoparticles (SLN) Based Hydrogel for Delivery of Etodolac: A Comparative Investigation on Dermal Pharmacokinetic on Rat Skin. Int. J. Pharm. Investigation, 2021;11 (2):220-4. 